\title{
Towards Functionality-Enhanced Devices: Controlling the Modes of Operation in Three-Independent-Gate Transistors
}

\author{
Pierre-Emmanuel Gaillardon, Jian Zhang, Michele De Marchi, Giovanni De Micheli \\ Integrated Systems Laboratory (LSI), EPFL, Switzerland \\ pierre-emmanuel.gaillardon@epfl.ch
}

\begin{abstract}
In this paper, we introduce the different modes of operation achievable with Three-Independent-Gate Field-Effect Transistors (TIGFETs) and report results on fabricated devices including: (i) the dynamic reconfiguration of the device polarity; (ii) the dynamic control of the threshold voltage; and (iii) the dynamic control of the subthreshold slope.
\end{abstract}

\section{INTRODUCTION}

As the semiconductor industry is approaching the ultimate limits of conventional silicon-based transistors, researchers are focusing their efforts to identify possible approaches that will enable the continuation of Moore's scaling laws. In parallel to the focus on scaling, an interesting approach consists in increasing the functionalities of individual devices [1]-[6].

In this paper, we review the different modes of operations enabled by Three-Independent-Gate Field-Effect Transistors (TIGFETs). We report on fabricated Schottky-barrier FET transistors featuring three independent gate-all-around electrodes. The two gate electrodes in proximity to the source and drain Schottky contacts are used to dynamically modulate the barrier heights and tune the operation of the transistor, while the third gate electrode, acting on the center region of the channel, serves as standard gate. The additional gate terminals bring extended functionalities to the device such as (i) the dynamic reconfiguration of the polarity ( $n$ - or $p$-type) [4]; (ii) the dynamic control of the threshold voltage $\left(\mathrm{V}_{\mathrm{T}}\right)$ that does not lead to any detriment of the on-state current [5]; and (iii) the dynamic control of the Subthreshold Slope (SS) allowing to trigger weak impact ionization and record an average SS of $6 \mathrm{mV} / \mathrm{dec}$ over 5 decades of current swings [6]. Devices with an enhanced set of functionalities are expected to extend the envelope of computation performance with high regularity and compactness, beyond the limits of scaling [7].

\section{ThreE-INDEPENDENT-GATE DEVICE OVERVIEW}

Fig. 1-a shows the structure of a TIGFET. The device exploits vertically-stacked nanowires as channel and metallic source and drain contacts. The channel electrostatics is controlled by three electrodes: The Polarity Gate at Source $\left(\mathrm{PG}_{\mathrm{S}}\right)$ and the Polarity Gate at Drain $\left(\mathrm{PG}_{\mathrm{D}}\right)$ modulate the Schottky barriers at source and drain; The Control Gate (CG) controls the potential barrier in the channel.

The TIGFET is fabricated with a dopant-free process on an SOI wafer [4]-[6]. The vertically-stacked nanowires are realized using a single Deep Reactive Ion Etching step [4]. Fig. 1-b shows an SEM image of the resulting silicon nanowire stack (length and diameter of $350 \mathrm{~nm}$ and $50 \mathrm{~nm}$, respectively). After a $15 \mathrm{~nm} \mathrm{SiO}{ }_{2}$ gate dielectric formation, two Gate-AllAround (GAA) $120 \mathrm{~nm}$ polysilicon structures are deposited to form $\mathrm{PG}_{S} / \mathrm{PG}_{D}$ and $\mathrm{CG}$ is subsequently self-aligned to them. Finally, NiSi silicide is formed on the source and drain pillars to create mid-gap Schottky barriers. Fig. 1-c and Fig. 1-d show a top SEM image and a cross-sectional view of the final structure.

\section{MODES OF OpERATIONS}

In a TIGFET, the control of carrier injection at the Schottky barriers by the PGs offers the capability to determine the operation modes of the device. In this section, we report on three different control mechanisms.

\section{A. Polarity Control}

In this case, we assume that the $\mathrm{PG}_{\mathrm{S}}$ and $\mathrm{PG}_{\mathrm{D}}$ terminals are connected together in a unique terminal called PG. Fig. 1-e presents a conceptual band diagram, showing the carriers involved in the device operation at different $\mathrm{CG}$ and PG biases. A positive PG bias enables electron conduction at the source and drain Schottky barriers, setting the device polarity to $n$ type, while a low PG bias leads to hole conduction and results in $p$-type behavior.

Polarity control on a fabricated device is observed in Fig. 1-f, where an increasing PG bias switches the device polarity from $p$ - to $n$-type. $I_{\text {on }} / I_{\text {off }}$ values ranging from $10^{6}$ to $10^{7}$ and subthreshold slopes of $64 \mathrm{mV} / \mathrm{dec}$ and $70 \mathrm{mV} / \mathrm{dec}$ were obtained, respectively, for the $p$ - and $n$-branches in the same physical device [4].

\section{B. Threshold Control}

In this second case, the two PG terminals are biased separately. The independent Schottky biasing enables an efficient $\mathrm{V}_{\mathrm{T}}$ control. Fig. 1-g and Fig. 1-h present the associated band diagrams for an $n$-type conduction.

Low- $\mathrm{V}_{\mathrm{T}} n$-type modes (Fig. 1-g) are similar to III-A. High$\mathrm{V}_{\mathrm{T}} n$-type modes (Fig. 1-h) are obtained when $\mathrm{PG}_{\mathrm{D}}=1, \mathrm{CG}=1$ and $\mathrm{PG}_{\mathrm{S}}$ controls the current flow. In the high- $\mathrm{V}_{\mathrm{T}}$ off state, the opposite band bending at the Schottky contacts prevents both electron and hole injection into the channel. Note that the low- $\mathrm{V}_{T}$ and high- $\mathrm{V}_{T}$ configurations share the same on state, reducing performance degradation.

The performances obtained on a fabricated device are shown in Fig. 1-i. A threshold difference of $0.86 \mathrm{~V}$ is observed. The high- $\mathrm{V}_{\mathrm{T}}$ off-state current reaches $1 \mathrm{pA}$ compared to $4.6 \mathrm{pA}$ 

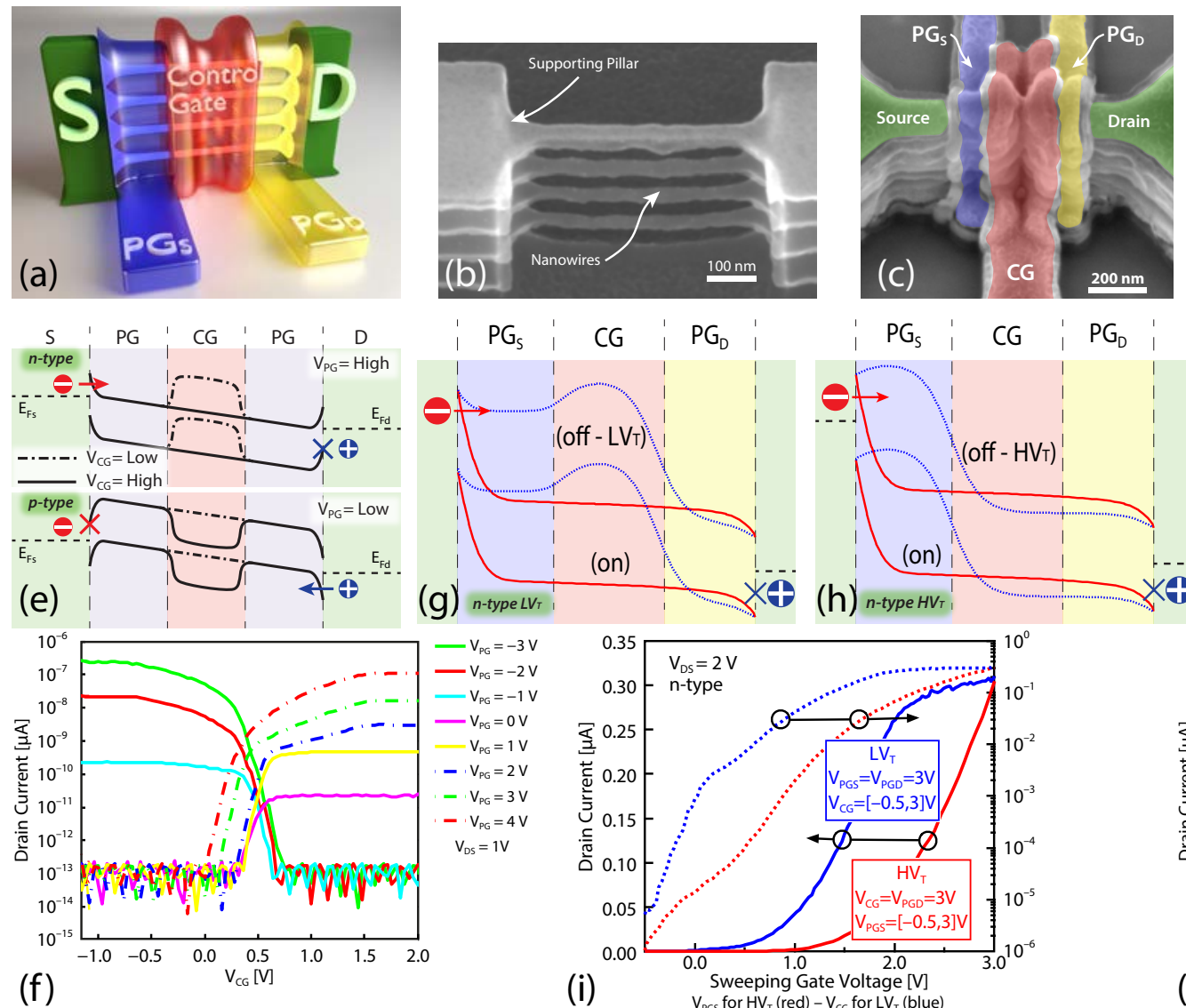

(i)
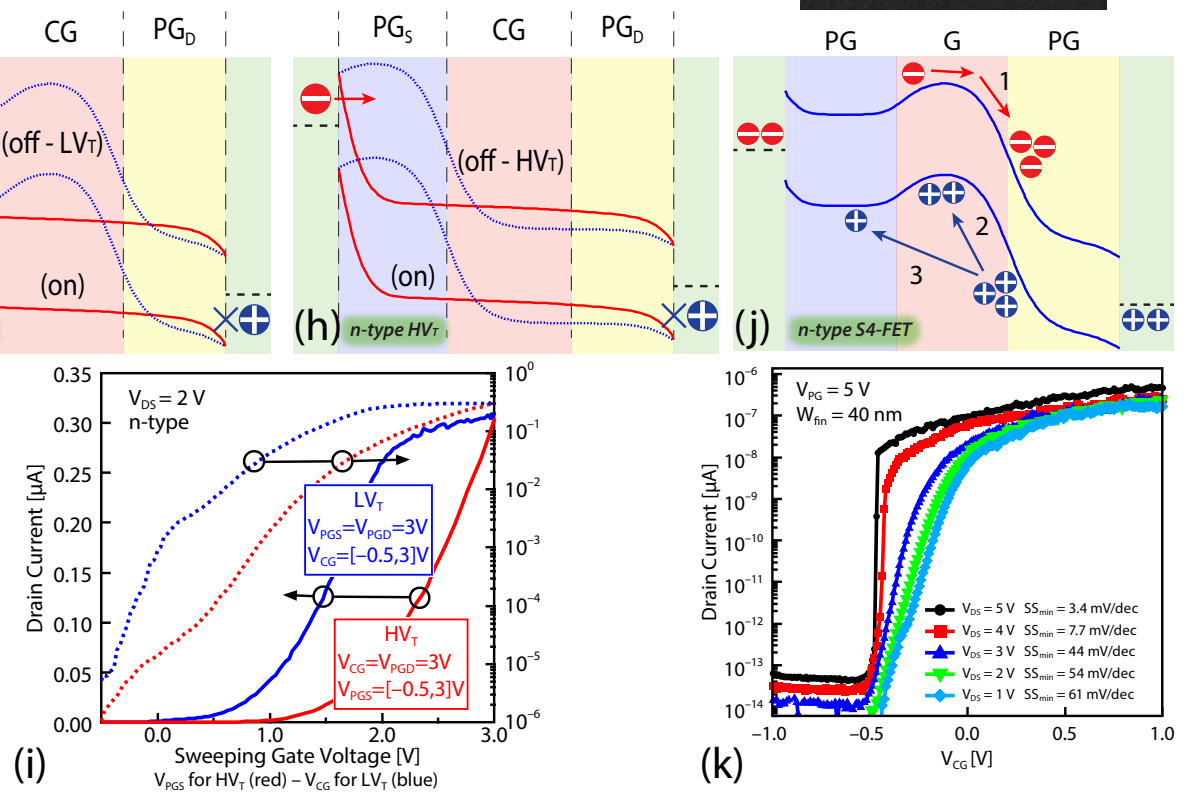

Fig. 1. Device fabrication: (a) Conceptual sketch of a TIGFET (b) Tilted SEM image of the fabricated vertically-stacked nanowire channel. (c) Tilted SEM image of the fabricated TIGFETs. (d) Cross-sectional SEM of the device at the overlapped CG and PG region. Polarity control: (e) Conceptual band diagrams of the device in the polarity control mode. Four cases are shown according the four combinations of high/low bias applied on the PG and CG terminals. Electron paths are shown with red arrows/crosses. Hole paths are shown with blue arrows/crosses. (f) Logarithmic $I_{D}-V_{C G}$ plot of the fabricated device at different $V_{\mathrm{PG}}$ biases. Threshold control: (g-h) Conceptual band diagrams of the device in the threshold control mode. Only $n$-type conditions are represented. Three combinations of $\left(\mathrm{PG}_{\mathrm{S}}, \mathrm{CG}, \mathrm{PG}_{\mathrm{D}}\right)$ are shown reflecting the on $(1,1,1)$, the $\mathrm{LV}_{\mathrm{T}}$ off $(1,0,1)$ and the $\mathrm{HV}_{\mathrm{T}}$ off $(0,1,1) n$-type states. (i) Measured linear and logarithmic $\mathrm{I}_{\mathrm{D}}-\mathrm{V}_{\mathrm{CG}} n$-type transfer characteristic showing the different $\mathrm{V}_{\mathrm{T}}$ conditions. Steep subthreshold control: (j) Conceptual band diagrams of the device in the S4-FET configuration, illustrating the mechanism of the impact-ionization-induced positive feedback during the on-transition in the $n$-type configuration. (k) Logarithmic $\mathrm{I}_{\mathrm{D}}-\mathrm{V}_{\mathrm{CG}}$ plot of the fabricated S4-FET device at different $V_{\mathrm{DS}}$ biases.

in low- $\mathrm{V}_{T}$. Full characterization of $n$ - and $p$-branches can be found in [5].

\section{Steep Slope Control}

In this third mode of operation, the two PGs are controlled together similarly to III-A. Here, we use the PGs to create a potential well under the gate and obtain a Super-Steep Subthreshold Slope device (S4-FET) [6]. The operation in $n$-type configuration is illustrated in Fig. 1-j. When electrons acquire enough energy, weak impact ionization is triggered and electron/hole pairs are generated (step 1). The generated holes accumulate in the potential well under the gate (step 2). This lowers the barrier and provides more electrons for impact ionization, thus establishing a positive feedback. During the transition, the energy band in the PG region is lowered (step 3 ), maintaining the potential well for the accumulation and improving the average SS over the subthreshold region.

Fig. 1-k shows the characteristics of the fabricated S4-FET [6]. Minimum SS of $3.4 \mathrm{mV} / \mathrm{dec}$ is achieved. An average SS of $6.0 \mathrm{mV} / \mathrm{dec}$ is observed for 5 decades of current. When decreasing $\mathrm{V}_{\mathrm{DS}}$, the impact ionization rate decreases, and the SS gradually degrades to $61 \mathrm{mV} / \mathrm{dec}$ at $V_{D S}=1 V$. Complete $n$-type and $p$-type characteristics are available in [6].

\section{CONCLUSIONS}

In this paper, we reported on the different modes of operation achievable in TIGFETs. Polarity, $\mathrm{V}_{\mathrm{T}}$ and steep subthreshold controls were demonstrated in fabricated devices showing a viable additional path to Moore's law for beyondCMOS computing.

\section{ACKNOWLEDGEMENTS}

This research was supported by ERC-2009-AdG-246810.

\section{REFERENCES}

[1] Y.-M. Lin et al., "High-performance carbon nanotube field-effect transistor with tunable polarities," IEEE Trans. Nanotechnology, vol. 4, pp. 481-489, 2005.

[2] N. Harada et al., "A polarity-controllable graphene inverter," Appl. Phys. Lett., vol. 96, 012102, 2010.

[3] A. Heinzig et al., "Reconfigurable silicon nanowire transistors," Nano Lett., vol. 12, pp. 119-124, 2012.

[4] M. De Marchi et al., "Polarity control in double-gate, gate-all-around vertically stacked silicon nanowire FETs," IEDM Tech. Dig., 2012.

[5] J. Zhang et al., "Polarity-Controllable Silicon Nanowire Transistors with Dual Threshold Voltages," Trans. on Electron Devices, pp. 339-342, 2014.

[6] J. Zhang et al., "A Schottky-barrier silicon FinFET with $6.0 \mathrm{mV} / \mathrm{dec}$ subthreshold slope over 5 decades of current," IEDM Tech. Dig., 2014.

[7] P.-E. Gaillardon et al., "Nanowire systems: technology and design," Phil. Trans. R. Soc. A, vol. 372, no. 20130102, 2014. 